\title{
Rethink of Concept Mass Based on Experiments of Chicken Embryo Egg
}

\author{
Jie Yang ${ }^{*}$ \\ School of Mechanics and Engineering, Southwest Jiaotong University, 610031 \\ Chengdu, Sichuan, China
}

\begin{abstract}
Mass is an attribute of physical matter in general. However, whether mass varies during death is an interesting problem. In the manuscript, experiments about chicken embryo eggs were design to check it. Chicken embryo egg was sealed in a cup with $440 \mathrm{ml}$, and monitored the mass variation during death process. The results show that for embryos of more than 14 days old, there were two turns on the mass time curve. The slope of line between two turns was larger than the one of the other, which means that extra mass loss happened between two turns. Experiments also show that for the chicken embryo of poor health, or early stage, mass loss was less than the health or late stage one. For the embryo of less than 14 days old, there was not clearly the second turn on the mass curve. Several kinds of experiments were designed and performed to confirm that instead of measuring error or gases permeation, the turns on the mass curve were a phenomenon related to death of embryo. For the embryo of more than 14 days old, the process of extra mass loss was most likely process of death, starting from the first turn, and ending at the second turn. The experiments hint that mass loss is a fundamental phenomenon in nature when a living being loses life; in addition to physical matter, mass is also a feature of life, which provides a new perspective to understand concept mass.
\end{abstract}

Key words: mass loss; death; chicken embryo egg; weigh; seal

\section{Introduction}

Mass maybe the most classical physical concept, which played a fundamental role in the great natural theories, such as law of universal gravitation, general relativity ${ }^{[1]}$ et.al.; and still push forward the progress of many frontier researches nowadays, such as, darkness matter ${ }^{[2]}$.

In common sense, mass is considered as an attribute of physical matter. However, whether life may possess mass either? In other words, whether mass varies during process of death? It is an interesting problem.

In 1907, Duncan declared that weight would lose when a man died; on the other hand, dogs had no mass loss during death ${ }^{[3]}$. The experiments were widely regarded as unscientific in scientific community ${ }^{[4][5]}$. Two flaws make experimental results unreliable, firstly, there was a significant error of measure in a no strictly closed environment; the other was small sample size, and only one in 6 tests met his hypothesis.

I've been attracted to thinking about mass change caused by life for years. After overcoming many sorts of difficulties, I selected a new animal model, designed new experiments and obtained credible evidences of mass change. In the manuscript, experiments and results are introduced and discussed in detail.

\section{Material and method}

Chicken embryo egg was chosen as animal model. The embryo eggs in the experiments belong to Chinese black bone chicken and lohman chicken with different days old.

In an experiment, an embryo egg was sealed in a plastic cup, and then measured by a balance; mass data of the egg and cup was send to a computer once a second during an experiment. The embryo died finally for lacking oxygen $\left(\mathrm{O}_{2}\right)$, which was exhausted by embryo breathing in the

\footnotetext{
* Corresponding author: yangchenjie@swjtu.cn
} 
sealed cup. Thereby, mass data of embryo egg during death process could be obtained.

A sealed cup in experiment, could prevent most of gas dismiss, make measurement precision higher than the one in an open environment; in addition, for chicken egg, embryo is confined to a closed egg shell, which made experiment easily performed.

In experiments, two types of analysis balance with $0.1 \mathrm{mg}$ resolution, Mattler Toledo@ ME104E and Shimadzhu@ AUW220 were adopted. The plastic cup, about $440 \mathrm{ml}$, is made of material polypropylene(P.P.). The temperature should keep stable during experiments, larger than $34^{\circ} \mathrm{C}$ and less than $38^{\circ} \mathrm{C}$, ranging less than $\pm 1{ }^{\circ} \mathrm{C}$. The main equipment used in experiments is shown in fig.1.

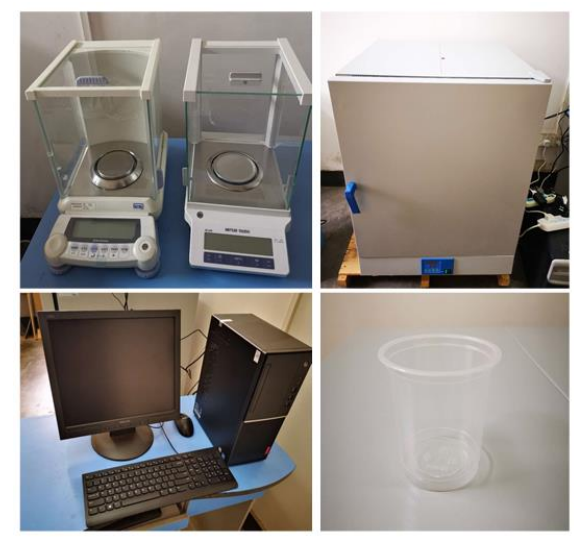

Fig.1. Main equipment in experiments: balance, incubator, computer and cup

\section{Results and discussion}

\section{Phenomenon of extra mass loss during process death.}

\subsection{A typical experiment and result}

Firstly, present typical result of chicken embryo egg of 17 days old, noting that 21 days needed until embryo hatching out. For comparison, experiment about water on the same conditions also show here. The two samples were sealed in cups by heat-sealed method respectively and weighed by balance Mattler Toledo ${ }^{@}$ ME104E. The curves of mass to time were shown in fig.2.

The results show the mass in two experiments all decreased at the beginning, about 40 minutes. This was not a real mass loss, but unstable measurement due to influence of initial load and heat, which was easily verified by experiment. In following discussion, this stage is omitted.

The mass of water lineally decreased with slope $4.34 * 10^{-4}$ milligrams per second (mg/s), as shown in fig.2b. The reason is that water would diffuse into the wall of cup, and permeate from cup. It's noted that the mass curve were linear, which means that the balance could obtain stable data of weight when measurement lasts long time, such as about 10 hours in the fig. $2 \mathrm{~b}$. 


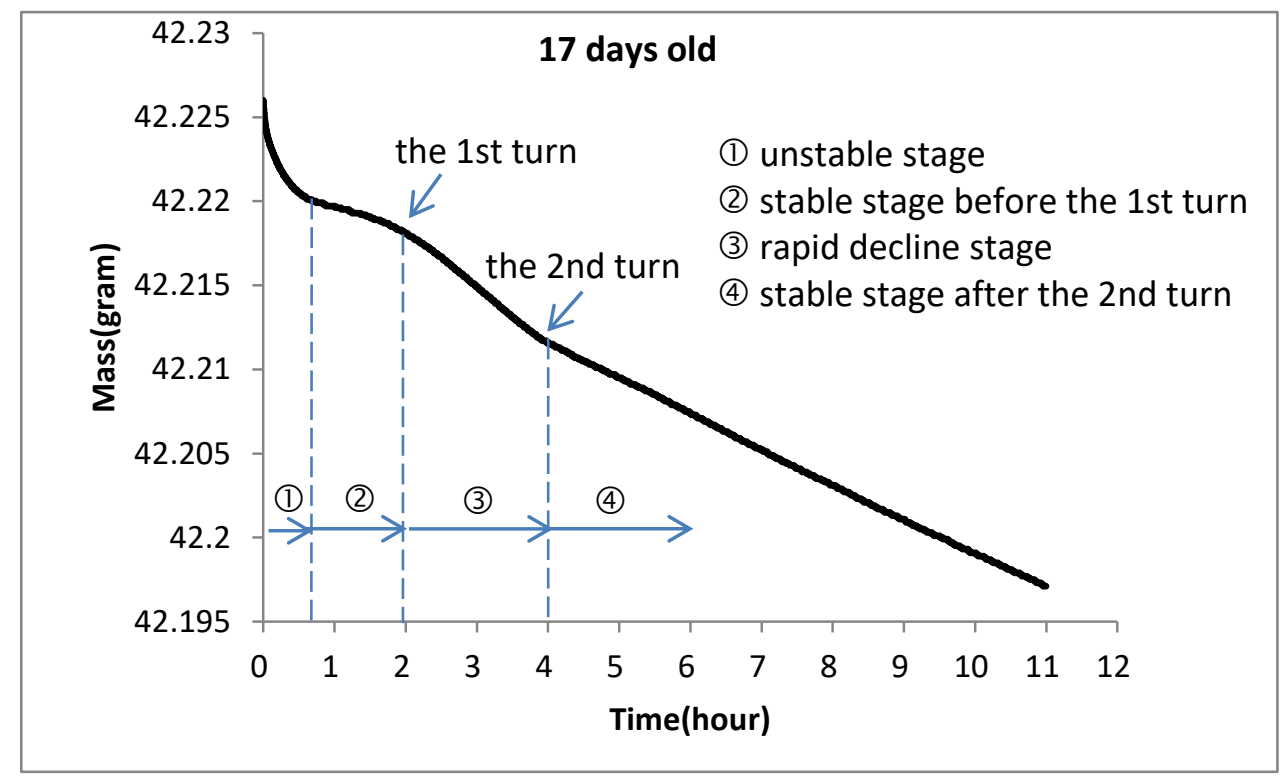

(a)

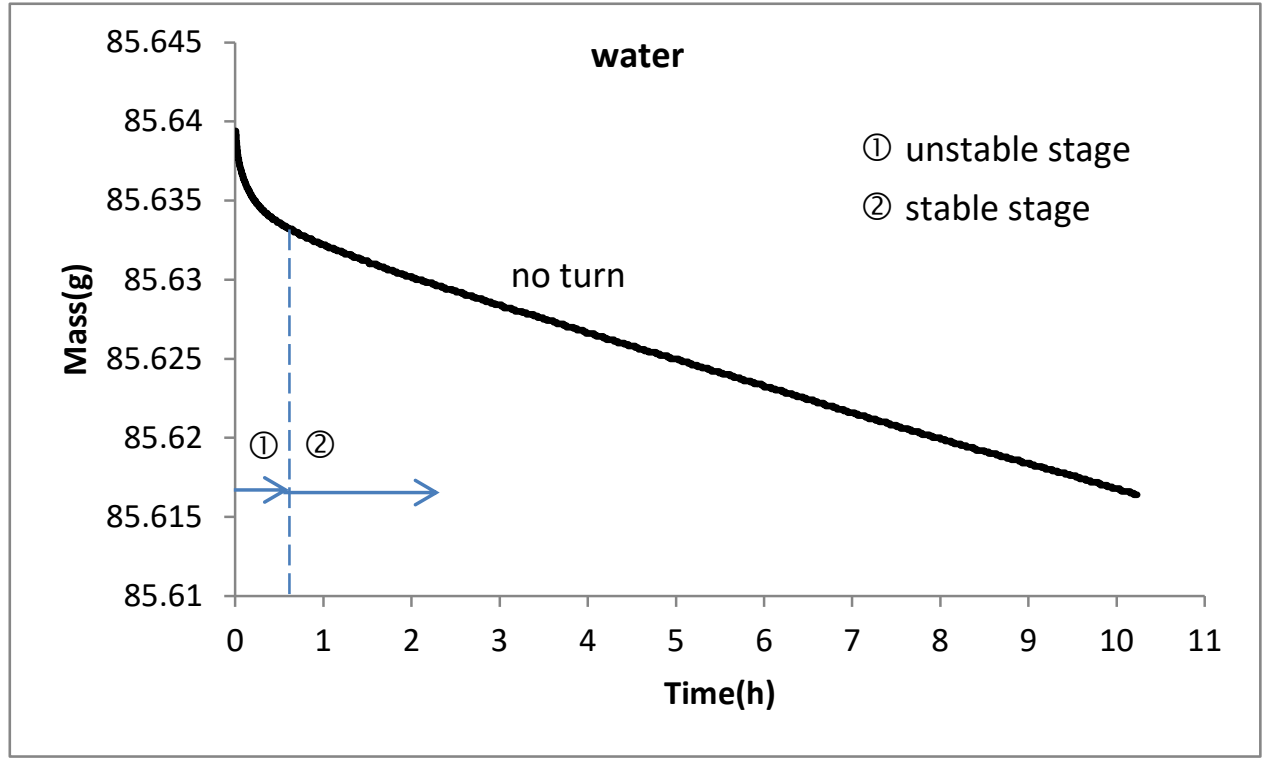

(b)

Fig.2. (a) Mass curve of 17 day old embryo egg sealed in a cup. Stable stage start at about 40 minutes, the first and second turn appeared about two hours and four hours respectively, the slope of line between two turns is larger than the slope before the first turn, and than the slope after the second turn, which mean that extra mass loss happen between two turns. (b)Water mass curve to time in a sealed cup, the curve after about 40 minutes lineally declined on the stable stage; there was no turn on stable stage.

However, for the embryo egg sealed in a cup, it is a different case. The experiment lasted 9 hour for ensuring death of the chicken embryo. The surprising phenomenon was that there were two turns on the mass curve after measurement kept stable, as shown in fig.2a. The first turn happened about at 2 hours; the second one was at about 4 hours. The slope between two turns, about $9.48 * 10^{-4} \mathrm{mg} / \mathrm{s}$, was larger than the slope before the first turn, about $3.81 * 10^{-4}$ $\mathrm{mg} / \mathrm{s}$; than the slope after the second turn, about $5.80 * 10^{-4} \mathrm{mg} / \mathrm{s}$, which means that extra mass loss happened comparing the other stages after measurement was stable. In detail, based 
on the stage after the second turn, extra mass loss, $2.65 \mathrm{mg}$ (notice: $(9.48-5.80) * 10^{-4} \mathrm{mg} / \mathrm{s} *$ $2 * 3600 \mathrm{~s}=2.65 \mathrm{mg}$ ), was happened during the stage between two turns. In the paper, this phenomenon is called "extra mass loss".

\subsection{General features of extra mass loss}

Experiments I performed in these years showed that, in general, the first turn was smooth; by comparison, the second one was sharp, even a turn point. The slope of line between two turns was always larger than one of the others.

Different individual of embryo egg owned different extra mass loss. There was no exactly same weight loss for the different individual. Two main influence factors are presented here as below:

Firstly, extra mass loss of embryo egg depended on the different incubating days. In general, the more incubating days, the more significant the second turn on the mass curve, and the larger extra mass loss was. When embryo egg was incubated less than 14 days, the second turn was unapparent on the mass curve, as shown in fig.3a.

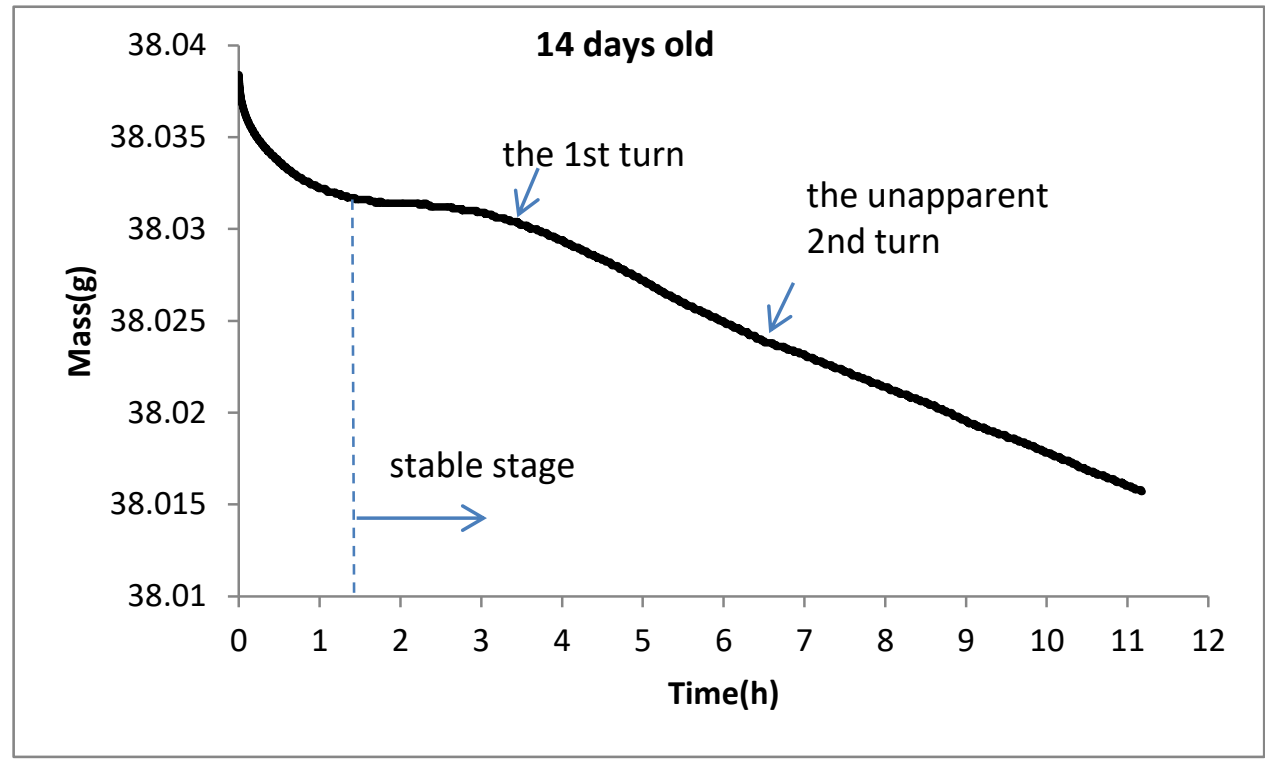

(a)

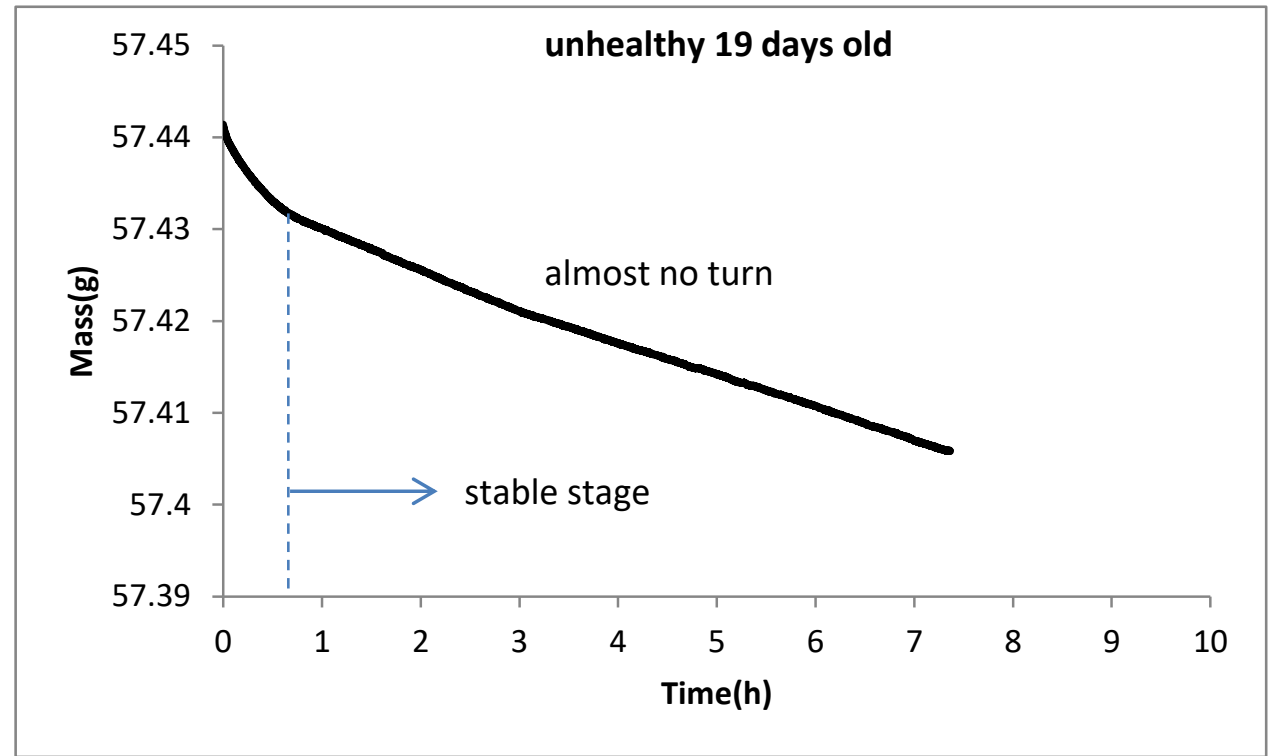

(b) 


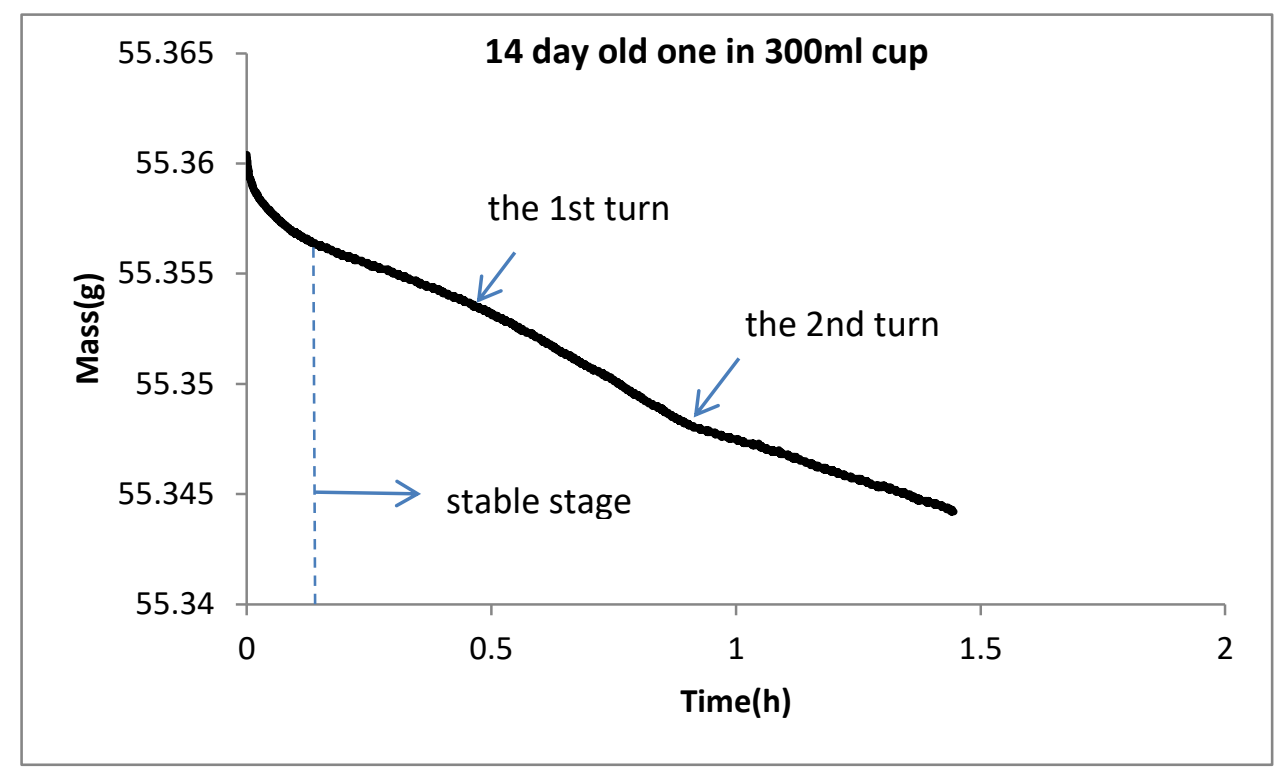

(c)

Fig.3. (a) Mass curve of 14 day old embryo egg sealed in a cup. The second turn was comparatively unapparent on the curve. (b) Mass curve of 19 day old unhealthy embryo egg sealed in a cup. There was almost no the first and the second turn on the curve. (c) Mass curve of 14 day old embryo egg sealed in $300 \mathrm{ml}$ cup. The second turn was more distinct on the curve than one in $440 \mathrm{ml}$ cup.

Secondly, although the mass curve of unhealthy egg owned turns, comparing a healthy one, the two turns were less apparent, and extra mass loss was smaller. Result of a 19 day old unhealthy embryo is shown in fig. $3 b$, the two turns almost disappeared on the mass curve.

In addition, significant level of the second turn on a mass curve was also influenced by volume of a cup, which was used to seal an egg. As shown in fig.3c, an embryo egg of 14 days old, sealed in a $300 \mathrm{ml}$ cup, the second turn on the mass curve was more distinct than the one in a $440 \mathrm{ml}$ cup, shown in fig.3a.

\section{Test verification}

\subsection{Real phenomenon of extra mass loss}

For verifying the phenomenon of extra mass loss is real, a series of experiments were designed and performed as below.

Firstly, instead of balance Mattler Toledo ${ }^{@}$ ME104E, balance SHIMADZHU ${ }^{@}$ AUW220 was adopted to measure; secondly, instead of thermal seal method, embryo egg was sealed by adhesive tape method; thirdly, to eliminate the influence of electromagnetic wave, embryo egg was wrapped up closely by aluminum foil before sealed in a cup, which would significantly weaken electromagnetic wave; fourthly, Instead of chicken embryo egg, an embryo egg of duck of 24 days old was measured, noting that incubating period of duck is 28 days. The results showed that phenomenon of two turns on mass curve, mass loss, were also observed in these experiments. The mass curve of duck's embryo egg is shown in fig.4.

In a conclusion, the extra mass loss is a real phenomenon, independent of balance, seal method, electromagnetic wave variation, and is strong likely a common phenomenon for general life during death. 


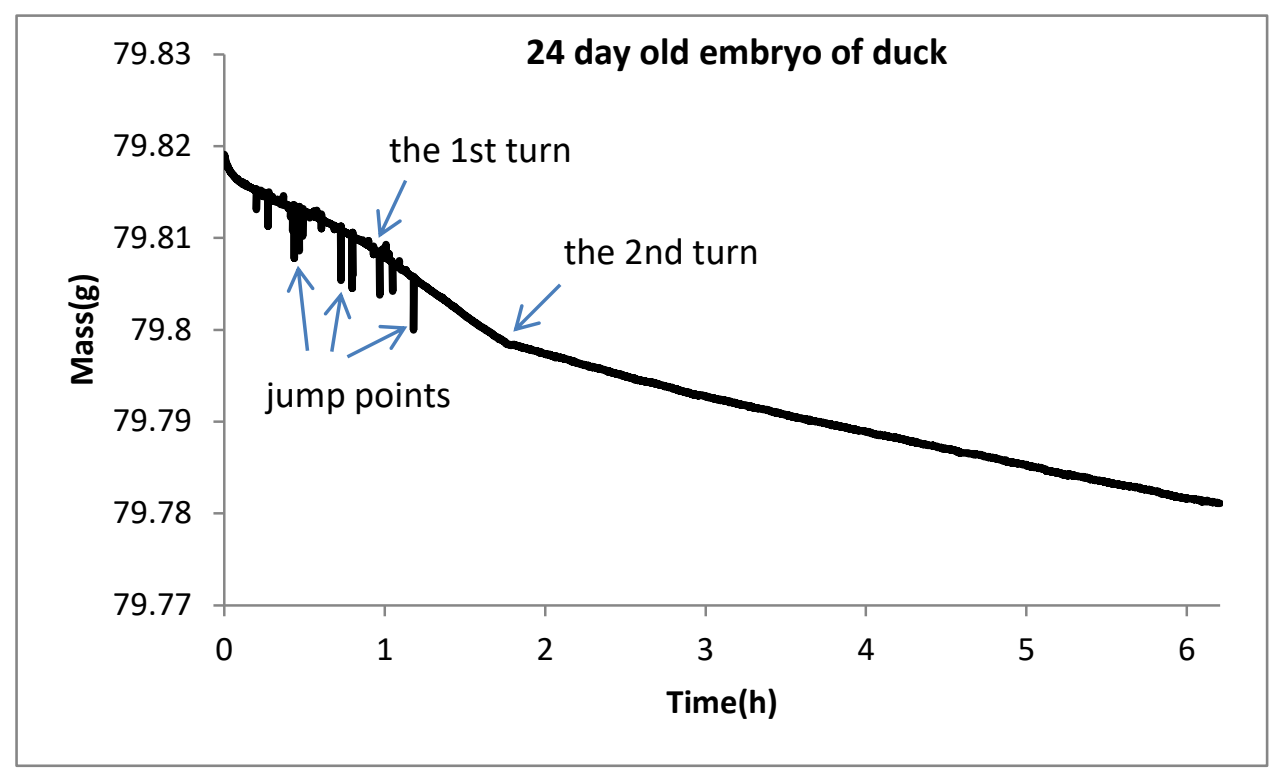

Fig.4. Mass curve of 24 day old egg of a duck. Movement of the embryo in egg shell could be detected by the balance, and presented as jump points on the curve, which happened before the second turn, and no jump point after it.

\subsection{Extra mass loss was irrelevant to ordinary gases permeating.}

With breathe, $\mathrm{O}_{2}$ would be exhausted, and carbon dioxide $\left(\mathrm{CO}_{2}\right)$ and water vapor, were filled in the cup gradually. Obviously, extra mass loss wasn't cause by $\mathrm{O}_{2}$ or nitrogen $\left(\mathrm{N}_{2}\right)$ escaping from cup. Experiments below were designed to confirm that extra mass loss wasn't caused by $\mathrm{CO}_{2}$ and water vapor either.

For eliminating interference of water vapor and $\mathrm{CO}_{2}$ produced by breathing in experiments, two nested cups were used. Firstly, an egg was put and sealed in an inner cup, and then sealed in an exterior one. Between two cups, it was full of water. Water could isolate water vapor and $\mathrm{CO}_{2}$ from the wall of the exterior cup. During experiment, permeation of water vapor and $\mathrm{CO}_{2}$ through the exterior cup would keep a constant on the whole.

As shown in fig.5, two turns always appeared in the curve, the same as the one without water. The experiment shows that extra mass loss also happened when influence of water vapor and $\mathrm{CO}_{2}$ was eliminated.

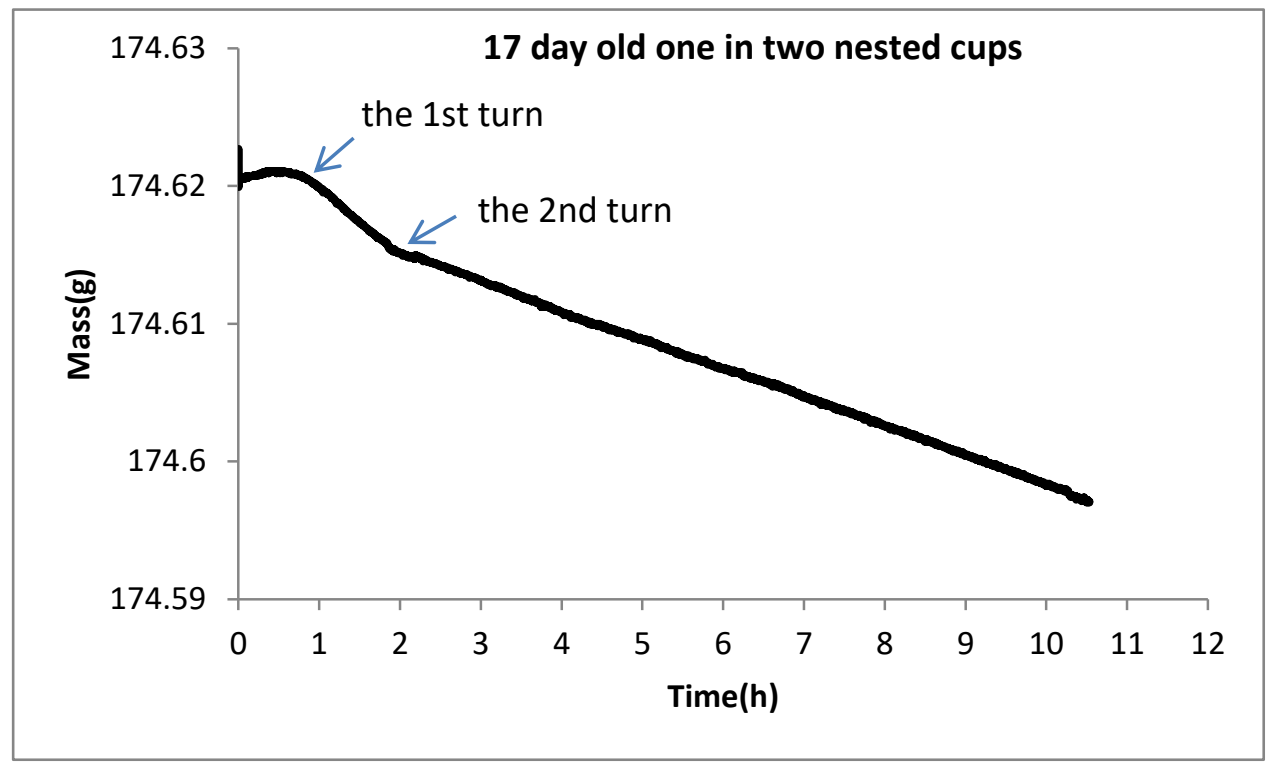


Fig.5. The mass curve of a 17 day old egg, which was sealed in two nested cups, full of water between them. The first and second turns were clearly on the mass curve.

In addition, when ordinary gases permeate from a closed plastic cup, it is impossible to form a turn like the second one on a mass curve. The second turn, being two lines with a sharp turn point, indicated that there was a kind of mass object escaping rapidly and completely from a plastic cup before the turn point. There was no ordinary gas satisfying this behavior.

In a conclusion, extra mass loss didn't caused by $\mathrm{CO}_{2}$ or water vapor, nor by ordinary gases, but was likely caused by death itself.

\section{Meanings of turns and mass loss.}

What happened on the turn points; what's the meaning of mass loss between the two points?

A phenomenon in the experiment below gave a clue about this problem. Some active embryo could make egg movement slightly, which would be detected by balance with solution $0.1 \mathrm{mg}$, as shown in fig.4. The movement of embryo happened before the second turn, and not appeared after it. On the other hand, an embryo egg couldn't be hatched out when it exceeding the second turn on a mass curve.

In addition, mass could keep a linear decline after second turn for a very long time, such as 4 days, the same as curve of an inanimate object.

These are strong possibility that the process of extra mass loss is the process of death, starting from the first turn, and ending at the second turn; the second one might be a critical point of complete death. After pass the second turn, the life couldn't be reversed.

\section{Deduction}

For a chicken, a healthy life own larger mass than an unhealthy one, than an unliving one ; an embryo of more days old own larger mass than a less day old one.

Although phenomenon of extra mass loss clearly emerged on chicken embryos beyond 14 days old during process of death, it's likely that extra mass loss might be found in the chicken embryo less than 14 days old if a balance was more precise and environment was more stable.

I surmise that mass loss during death is general phenomenon in nature; in other words, life own mass.

\section{Conclusions}

First of all, it's necessary to emphasize that two concepts, life and mass, are involved in the manuscript: Life is a basic phenomenon of nature, mass is a classical concept in physics. The relationship between the two concepts is a key point of the manuscript.

From the view of physics, concept mass is an attribute of physical matter. Mass was adopted to measure inertia and gravitation in Newtonian mechanics; and was further developed by Einstein, who unified mass and energy in $1905^{[6]}$.

However, there is still a long way to go before mass could be understood completely. For example, dark matter has mystified scientists for decades because classic theory of mass met huge difficulties: observable mass of some galaxies couldn't catch celestial bodies at high velocity on their orbits ${ }^{[2]}$, which shows that there are still unknown territories about mass need to be explored.

Experiments in the manuscript show that mass variation is likely affected by state of life. This is new phenomenon about mass. It hints that life might own mass, which provides a new perspective to understand mass and life. 
I deem that mass owned by life should be investigated comprehensively, or we might miss new approaches and theories of mass, which are undoubtedly valuable to understand nature.

At last, experiments in the manuscript are clear, simple, repeatable and low cost. It is time for more researchers to check the results.

\section{Reference}

1. Einstein A.(1916). Relativity: The Special and General Theory (Translation 1920), New York:H.Holt and Company.

2. J. C. Kapteyn(1922). First Attempt at a Theory of the Arrangment and Motion of the Sidereal System. The Astrophysical Journal,55:302

3. Mac Dougall, Duncan(1907). The Soul: Hypothesis Concerning Soul Substance Together with Experimental Evidence of the Existence of Such Substance. American Medicine. 2:240-243.

4. Kruszelnicki, Karl (2006). Great Mythconceptions: The Science Behind the Myths. Andrews McMeel Publishing. 199-201. ISBN 9780740753640.

5. Wiseman, Richard (2011). Paranormality: Why We see What Isn't There. Macmillan. 32-34. ISBN 978-1743038383.

6. A. Einstein(1905). Does the Inertia of a Body Depend on its Energy Content? Annals Physics. 17:891-893. 